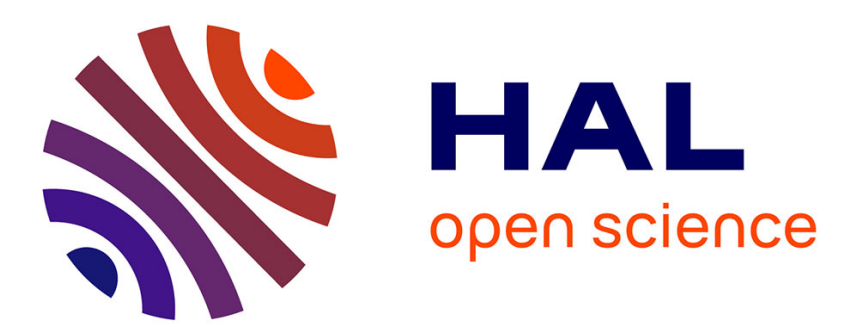

\title{
A formal $\Gamma$-convergence approach for the detection of points in 2-D images
}

\author{
Daniele Graziani, Laure Blanc-Féraud, Gilles Aubert
}

\section{To cite this version:}

Daniele Graziani, Laure Blanc-Féraud, Gilles Aubert. A formal $\Gamma$-convergence approach for the detection of points in 2-D images. [Research Report] RR-7038, INRIA. 2009, pp.25. inria-00418526

\section{HAL Id: inria-00418526 \\ https://hal.inria.fr/inria-00418526}

Submitted on 19 Sep 2009

HAL is a multi-disciplinary open access archive for the deposit and dissemination of scientific research documents, whether they are published or not. The documents may come from teaching and research institutions in France or abroad, or from public or private research centers.
L'archive ouverte pluridisciplinaire HAL, est destinée au dépôt et à la diffusion de documents scientifiques de niveau recherche, publiés ou non, émanant des établissements d'enseignement et de recherche français ou étrangers, des laboratoires publics ou privés. 
1 INSTITUT NATIONAL DE RECHERCHE EN INFORMATIQUE ET EN AUTOMATIQUE

\section{A formal $\Gamma$-convergence approach for the detection of points in 2-D images.}

Daniele Graziani, Laure BLANC-FÉRAUD, Gilles Aubert

$\mathbf{N}^{\circ} \mathbf{7 0 3 8}$

2009

Thème COG

apport

de recherche 



\title{
3 A formal $\Gamma$-convergence approach for the detection of points in 2-D images.
}

5

\author{
Daniele Grazian‡, Laure BLANC-FÉRAUD*, Gilles Aubert \\ Thème COG - Systèmes cognitifs \\ Projet ARIANA \\ Rapport de recherche $n^{\circ} 7038-2009$ - xxiv pages
}

9 Abstract: We propose a new variational model to isolate points in a 2-dimensional image.

\begin{abstract}
To this purpose we introduce a suitable functional whose minimizers are given by the points we want to detect. In order to provide numerical experiments we replace this energy with a sequence of a more treatable functionals by means of the notion of $\Gamma$-convergence.
\end{abstract}

Key-words: points detection, curvature-depending functionals, divergence-measure fields, $\Gamma$-convergence, biological 2-D images.

* ARIANA Project-team, CNRS/INRIA/UNSA, 2004 Route des lucioles-BP93, 06902 Sophia-Antipolis Cedex, France

${ }^{\dagger}$ LABORATOIRE J.A. DIEUDONNÉ Université de Nice SOPHIA ANTIPOLIS, parc valrose 06108 Nice CEDEX 2, FRANCE. 
${ }_{15}$ A $\Gamma$-convergence approach for the detection of points in

16 2-D images.

17 Résumé :

18 Mots-clés : 
19 Contents

20

21

2 Preliminaries

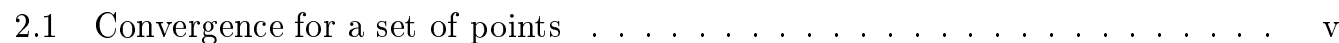

2.2 Distributional divergence $\ldots \ldots \ldots \ldots \ldots \ldots$ v

2.3 The Dirichlet problem with measure data $\ldots \ldots \ldots \ldots$ vi

3 Existence result vii

4 Г-convergence $\mathrm{x}$

4.1 Modica Mortola's approach . . . . . . . . . . . . . . . . . . . . x

4.2 De Giorgi's conjecture . . . . . . . . . . . . . . . . . . . . . $\mathrm{x}$

5 The approximating functionals xii

6 Detection xv

6.1 Discretization ...................... . . . . . . . . .

6.2 Discretization in time $\ldots \ldots \ldots \ldots \ldots$. . . . . . . . . . . . . . . . .

7 Computer examples xvii

7.1 Parameter settings . . . . . . . . . . . . . . . . xvii

7.2 Commentaries . . . . . . . . . . . . . . . . . . . . . xvii

8 Conclusion $\quad x x i i$

References $\quad$ xxiii 
Daniele Graziani et al.

\section{Introduction}

Detecting fine structures, like points or curves in two or three dimensional images respectively, is an important issue in image analysis. In biological images a point may represent a viral particle whose visibility is compromised by the presence of other structures like cell membranes or some noise.

From a variational point of view the problem of isolating points is a difficult task, since it is not clear how these singularities must be classified in terms of some differential operator. Indeed, since these are usually defined as discontinuity without jump, we cannot use the gradient operator as in the classical problem of contours detection. As a consequence the functional framework we have to deal with may be not clear.

One possible strategy to overcome this obstacle is considering these kinds of pathology as a $k$-codimension object, meaning that they should be regarded as a singularity of a map $U: \mathbb{R}^{k+m} \rightarrow \mathbb{R}^{k}$, with $k \geq 2$ and $m \geq 0$ (see [7] for a complete survey on this subject). So that the detecting point case corresponds to the case $k=2$ and $m=0$.

In this direction, in [5], the authors have suggested a variational approach based on the theory of Ginzburg-Landau systems. In their work the isolated points in 2-D images are regarded as the topological singularities of a map $U: \mathbb{R}^{2} \rightarrow \mathbb{S}^{1}$, where $\mathbb{S}^{1}$ is a unit sphere of $\mathbb{R}^{2}$. So that it is crucial to construct, starting from the initial image $I: \mathbb{R}^{2} \rightarrow \mathbb{R}$, an initial vector field $U_{0}: \mathbb{R}^{2} \rightarrow \mathbb{S}^{1}$ with a topological singularity of degree 1 at points in the initial image $I$ where the intensity is high. How to do this in a rigorous way, it is a subject of a current investigation.

Here our purpose is to provide a lighter variational formulation, in which the singularity in the image is directly given in terms of a proper differential operator defined on vector fields. Another important difference is that in 5 points and curves are detected both as singularities, while in the present paper our aim is to isolate from the initial image points and at same time remove any other singularities like curves precisely. In our model $\Omega \subset \mathbb{R}^{2}$ is an open set and represents the image domain.

In order to detect the singularities of the image, we have to find a functional space whose elements generate, in a suitable sense, a measure concentrated on points. Such a space is $\mathcal{D M}^{p}(\Omega)$ introduced in [4] where $1<p<2$, the space of vector fields $U: \Omega \rightarrow \mathbb{R}^{2}$ whose distributional divergence is a Radon measure (see Subsection 2.2 for definitions and examples). The restriction $1<p<2$ is due to the inizialization (see subsection 2.3).

Unfortunately even if we are capable of constructing an initial vector field $U_{0}$ (see below for such a construction) belonging to the space $\mathcal{D} \mathcal{M}^{p}(\Omega)$, its singular set could contains several structures we want to remove from the original image like for instance curve or some noise. Hence, after the initialization we have to clear away all the structures we are not interested in by building up, starting from the initial data $U_{0}$, a new vector field $U$ whose singularities are given by the points of the image $I$ we want to isolate.

Thus, from one hand we have to force the concentration set of the distributional divergence of $U_{0}$ to contain only the points we want to catch, and on the other hand we have to regularize the initial data $U_{0}$ outside the points of singularities. To this end we propose to minimize an energy involving a competition between a divergence term and the counting 
so

Hausdorff measure $\mathcal{H}^{0}$. More precisely the energy is the following

$$
\int_{\Omega \backslash P}|\operatorname{div} U|^{2}+\lambda \int_{\Omega}\left|U-U_{0}\right|^{p}+\mathcal{H}^{0}(P) .
$$

where $U \in L^{p, 2}(\operatorname{div} ; \Omega \backslash P)$ is the space of $L^{p}$-vector fields whose distributional divergence belongs to $L^{2}(\Omega \backslash P), P$ is the atomic set we want to target and $\lambda$ is a positive weight. The first integral forces $U$ to be regular outside $P$, while the term $\mathcal{H}^{0}(P)$ penalizes the presence of singular curves in the image.

From a practical point of view, this choice allows us to work with a first order differential operator and permits us to formulate the minimization problem in a common functional framework.

Clearly for inizialiting the minimization process we need to construct from the initial image, a vector field $U_{0}$ belonging to $\mathcal{D} \mathcal{M}^{p}(\Omega)$. Such a vector field can be provided by the gradient of weak solution of the classical Dirichlet problem with measure data.

$$
\begin{cases}\Delta f=I & \text { on } \Omega \\ f=0 & \text { on } \partial \Omega .\end{cases}
$$

In order to provide computer examples, we must approximate functional (1) by means of a sequence of more convenient functionals.

The approximation, we suggest in this paper, is based on the so called $\Gamma$-convergence, the notion of variational convergence introduced by De Giorgi (see [13, 14). This theory is designed to approximate a variational problem by a sequence of different variational problems. The most important feature of the $\Gamma$-convergence relies on the fact that it implies the convergence of minimizers of the approximating functionals to those of the limiting functional. In this work we suggest a possible $\Gamma$-convergence approach for the detection of points. By the way we stress out that the $\Gamma$ convergence result is only conjectured in this paper, whose purpose is to test a new variational method from an experimental point of view. For a rigourous variational approximation in a particular case, we refer the reader to 6.

Classically variational approximation techniques such as $\Gamma$-convergence or continuation method (see [2, 3] and [20] respectively) have been succesfully employed in image and signal processing.

For instance in ([2, 3]) Ambrosio and Tortorelli have proven that the classical MumfordShah's functional for detecting 1-dimensional smooth boundaries, can be approximated by a sequence of elliptic functionals that are numerically more treatable.

The main difficulty here is related to the presence of a codimension 2 object, which is not a contour: the set $P$. In order to obtain a variational approximation close to the one provided in $([2,3])$, the crucial step is then to replace the term $\mathcal{H}^{0}(P)$ of functional (10) by a more handy, from a variational point of view, functional involving a smooth boundary and his perimeter given by the 1-dimensional Hausdorff measure $\mathcal{H}^{1}$. Following some suggestion from the paper of Braides and Malchiodi and Braides and March (see 9 [0]) such a functional 
is given by:

$$
G_{\beta_{\varepsilon}}(D)=\frac{1}{4 \pi} \int_{\partial D}\left(\frac{1}{\beta_{\varepsilon}}+\beta_{\varepsilon} \kappa^{2}(x)\right) d \mathcal{H}^{1}(x),
$$

where $D$ is a proper regular set containing the atomic set $P, \kappa$ is the curvature of its boundary, the constant $\frac{1}{4 \pi}$ is a normalization factor, and $\beta_{\varepsilon}$ infinitesimal as $\varepsilon \rightarrow 0$. Roughly speaking the minima of this functional are achieved on the union of balls of small radius, so that when $\beta_{\varepsilon} \rightarrow 0$ the functional shrinks to the atomic measure $\mathcal{H}^{0}(P)$. On the other hand the introduction of a curvature term requires a non trivial and convenient, for a numerical point of view, approximation of the curvature-dependent functional. Such an approximation is based on a celebrated conjecture due to De Giorgi (see 12]). By means of this argument it is possible to substitute the curvature-depending functional with an integral functional involving the Laplacian operator of smooth functions. Then it remains to approximate the $\mathcal{H}^{1}$-measure and this can be done by retrieving a classical gradient approach used in 15. 16. This strategy allows to deal with a functional whose Euler-Lagrange equations can be discretized. A simple and intuitive explanation of the construction of the complete approximating functionals will be given in section 3 .

The paper is organized as follows: section 2 is intended to remind the reader of mathematical tools useful in the following. In section 3 we address the existence result for the functional $F(U, P)$ defined in (1). In section 4 we state the two well-known $\Gamma$-convergence results we need in the sequel. In section 5 we build in a formal way the approximating sequence. In section 6 we present the discrete model and the detection strategy. Finally the last section is devoted to some computer examples. 
Definition 2.3. For $U \in L^{p}\left(\Omega ; \mathbb{R}^{2}\right), 1 \leq p \leq+\infty$, set

$$
|\operatorname{div} \mathrm{U}|(\Omega):=\sup \left\{\int_{\Omega} U \cdot \nabla \varphi d x d y: \varphi \in C_{0}^{1}(\Omega),|\varphi| \leq 1\right\} .
$$

We say that $U$ is an $L^{p}$-divergence measure field, i.e. $U \in \mathcal{D} \mathcal{M}^{p}(\Omega)$ if

$$
\|U\|_{\mathcal{D M}^{p}(\Omega)}:=\|U\|_{L^{p}\left(\Omega ; \mathbb{R}^{2}\right)}+|\operatorname{div} \mathrm{U}|(\Omega)<+\infty .
$$


Remark 2.1. If $U \in \mathcal{D} \mathcal{M}^{p}(\Omega)$ then via Riesz Theorem it is possible to represent the distributional divergence of $U$ by a Radon measure. More precisely there exists a Radon measure $\mu$ such that for every $\varphi \in C_{0}^{1}(\Omega)$ the following equality holds:

$$
\int_{\Omega} U \cdot \nabla \varphi d x d y=-\int_{\Omega} \varphi d \mu .
$$

For instance the field $U(x, y)=\left(\frac{x}{x^{2}+y^{2}}, \frac{y}{x^{2}+y^{2}}\right)$ belongs to $\mathcal{D} \mathcal{M}_{\text {loc }}^{1}\left(\mathbb{R}^{2}\right)$ and its divergence measure is given by $-2 \pi \delta_{0}$, where $\delta_{0}$ is the Dirac mass.

Such a result can be proven by approximation. Let us define the following map:

$$
U_{\varepsilon}(x, y):= \begin{cases}U(x, y) & \text { if }|x| \geq \varepsilon \\ \left(\frac{x}{\varepsilon^{2}}, \frac{y}{\varepsilon^{2}}\right) & \text { if }|x|<\varepsilon .\end{cases}
$$

It is not difficult to check that $u_{\varepsilon}$ is Lipschitz-map with divergence given by $\frac{2}{\varepsilon^{2}} \chi_{B(0, \varepsilon)}$.

Then for every test function $\varphi \in C_{0}^{1}\left(\mathbb{R}^{2}\right)$ we have

$$
\int U_{\varepsilon} \cdot \nabla \varphi d x d y=-\int \frac{2}{\varepsilon^{2}} \chi_{B(0, \varepsilon)} \varphi d x d y .
$$

By applying the change of variables $x=\frac{x_{1}}{\varepsilon}, y=\frac{y_{1}}{\varepsilon}$ we obtain

$$
\int U_{\varepsilon} \cdot \nabla \varphi d x d y=-2 \int \chi_{B(0,1)} \varphi\left(\frac{x_{1}}{\varepsilon}, \frac{y_{1}}{\varepsilon}\right) d x_{1} d y_{1}
$$

so that, letting $\varepsilon \rightarrow 0$, by the dominated convergence theorem we obtain

$$
\int_{\Omega} U \cdot \nabla \varphi d x d y=-2 \pi \varphi(0,0)=-2 \pi \int_{\Omega} \varphi d \delta_{0}
$$

\subsection{The Dirichlet problem with measure data}

For the initialization of our algorithm we must build a vector field $U_{0}$ which should be such that its divergence is singular on points of the image $I$. Therefore we will use the gradient of the solution of the following Dirichlet problem (applied with $\mu=I$ )

$$
\begin{cases}\Delta f=\mu & \text { on } \Omega \\ f=0 & \text { on } \partial \Omega\end{cases}
$$

where $\mu$ is a Radon measure.

Classical results (see [19]) guarantee the existence of a unique solution of problem (4). Concerning the regularity it is known then $f \in W^{1, p}(\Omega)$ with $p<2$. 
167

184

\section{Existence result}

In this section we show the existence of a minimizing pair $(U, P)$ for the functional $F$ defined in (1)

Our argument needs two steps (see also 17 for a similar approach to minimize the classical Mumford-Shah's functional). The first one consists in proving the existence a minimizer of the functional (1) when the set $P$ is fixed.

To this aim we adopte the following notation:

$$
F(U)=F(\cdot, P)=\int_{\Omega \backslash P}|\operatorname{div} U|^{2} d x d y+\lambda \int_{\Omega}\left|U-U_{0}\right|^{p} d x d y+\mathcal{H}^{0}(P) .
$$

Theorem 3.1. For every set $P$ there exists a unique minimizer $U_{P} \in L^{p, 2}(\Omega \backslash P$; div) of the functional (5).

Proof. Let $U_{n}$ be a minimizing sequence. Then we have the following bound

$$
F\left(U_{n}\right) \leq M .
$$

From the bound (6) and the classical inequality:

$$
\left\|U_{n}\right\|_{L^{p}(\Omega \backslash P)}^{p} \leq 2^{p}\left\|U_{n}-U_{0}\right\|_{L^{p}(\Omega \backslash P)}^{p}+\left\|U_{0}\right\|_{L^{p}(\Omega \backslash P)}^{p}
$$

it follows that

$$
\left\|U_{n}\right\|_{L^{p}(\Omega \backslash P)}^{p} \leq M+\left\|U_{0}\right\|_{L^{p}(\Omega \backslash P)}^{p}:=C .
$$

Moreoveor we also have:

$$
\left\|\operatorname{div} U_{n}\right\|_{L^{2}(\Omega \backslash P)}^{2} \leq F\left(U_{n}\right) \leq M
$$

so that, up to subsequences, we obtain

$$
\begin{cases}U_{n} \rightarrow U_{P} & \text { in } L^{p}(\Omega \backslash P) \\ \operatorname{div} U_{n} \rightarrow \operatorname{div} U_{P} & \text { in } L^{2}(\Omega \backslash P) .\end{cases}
$$

Therefore we can conclude that $U_{n}$ weakly converges in $L^{p, 2}(\Omega \backslash P$; div) to a vector field $U_{P} \in L^{p, 2}(\Omega \backslash P ; \operatorname{div})$.

Then we have thanks to semicontinuity properties of the $L^{p}$-norm with respect to the weak convergence:

$$
\inf _{U} F(U) \leq F\left(U_{P}\right) \leq \liminf _{n \rightarrow+\infty} F\left(U_{n}\right)=\inf _{U} F(U) .
$$

Finally the strong convexity of functional (5) gives the uniqueness of the minimizer $U$

Once we obtained the existence of the minimizer $U_{P}$ for every set $P$ fixed, we focus on the following functional.

$$
E(P):=F\left(U_{P}, P\right)=\int_{\Omega \backslash P}\left|\operatorname{div} U_{P}\right|^{2} d x d y+\lambda \int_{\Omega}\left|U_{P}-U_{0}\right|^{p} d x d y+\mathcal{H}^{0}(P) .
$$


Lemma 3.1. Assume that a sequence of finite sets of points $\left\{P_{n}\right\} \subset \bar{\Omega}$ converges to a finite set of point $P \subset \bar{\Omega}$. Then

$$
E(P) \leq \liminf _{h \rightarrow \infty} E\left(P_{n}\right)
$$

Proof. Let us set $U_{n}=U_{P_{n}}$, we can assume that $U_{n}$ and $\operatorname{div} U_{n}$ are both defined on all of $\Omega$ in the sense explained above. The sequence $U_{n}$ is bounded in $L^{p}(\Omega)$. Indeed, by taking into account that $U_{n}$ is a minimizer of the functional (5),

$$
\left\|U_{n}\right\|_{L^{p}(\Omega)}^{p} \leq 2^{p}\left\|U_{n}-U_{0}\right\|_{L^{p}(\Omega)}^{p}+\left\|U_{0}\right\|_{L^{p}(\Omega)}^{p} \leq 2^{p} F(0)+\left\|U_{0}\right\|_{L^{p}(\Omega)}^{p}=\left\|U_{0}\right\|_{L^{p}(\Omega)}^{p}\left(2^{p}+1\right) .
$$

In the same way one can show that the sequence $\operatorname{div} U_{n}$ is bounded in $L^{2}(\Omega)$ So that, up to subequences, we may assume

$$
\begin{cases}U_{n} \rightarrow U & \text { in } L^{p}(\Omega) \\ \operatorname{div} U_{n} \rightarrow V & \text { in } L^{2}(\Omega)\end{cases}
$$

We claim that $\operatorname{div} U=V$ in $\Omega \backslash P$. In fact, take any test function $\varphi$ with support in $\Omega \backslash P$, then since $P_{n} \rightarrow P$, we have for $n$ large enough

$$
\operatorname{supp}(\varphi) \subset \Omega \backslash P_{n}
$$

and, consequently,

$$
\int_{\operatorname{supp}(\varphi)} U_{n} \nabla \varphi d x=-\int_{\operatorname{supp}(\varphi)} \operatorname{div} U_{n} \varphi d x .
$$

Therefore, by taking the weak limit by (9) we get

$$
\int_{\operatorname{supp}(\varphi)} U \nabla \varphi d x=-\int_{\operatorname{supp}(\varphi)} V \varphi d x .
$$

Then since the test function $\varphi$ is arbitrary, we can conclude that $\operatorname{div} U=V$ on $\Omega \backslash P$.

The thesis follows because, from the lower semicontinuity of the $L^{p}$-norm and Lemma 2.2

$$
E(P) \leq E(U, P) \leq \liminf _{n} E\left(U_{n}, P_{n}\right)
$$

We are now in position of proving the main result of this section.

193 Theorem 3.2. There exist a minimizer $(U, P)$ of the functional $F$, with $U \in L^{p, 2}(\operatorname{div} ; \Omega)$ and $P \subset \Omega$ a finite set of points. 
195 Proof. For every $P$ let $U_{P}$ a minimizer for the functional $F$, whose existence is guaranteed 196 by Theorem 3.1

Then we focus on the the functional $E(P)=F\left(U_{P}, P\right)$ and we take a minimizing sequence $\left\{P_{n}\right\}$. Then by Lemma 2.1] we have (up to a subsequences) that $P_{n} \rightarrow \bar{P} \subset \bar{\Omega}$ and $U_{P_{n}} \rightarrow U_{\bar{P}}$. By Lemma 3.1 we get

$$
E(\bar{P}) \leq \liminf _{n \rightarrow+\infty} E\left(P_{n}\right)
$$

197 Therefore

$$
\inf _{(U, P)} F(U, P) \leq F\left(U_{\bar{P}}, \bar{P}\right) \leq \liminf _{n \rightarrow+\infty} E\left(P_{n}\right) \leq \liminf _{h \rightarrow+\infty} F\left(U_{n}, P_{n}\right)=\inf _{P} F\left(U_{P}, P\right) \leq F(U, P),
$$

Now set $\tilde{P}:=\bar{P} \backslash \partial \Omega$. Since for every $P, U_{P}$ is a minimizer we get from (10)

$$
F\left(U_{\bar{P}}, \tilde{P}\right) \leq F\left(U_{\bar{P}}, \bar{P}\right) \leq F\left(U_{P}, P\right) \leq F(U, P),
$$

for every $(U, P)$. Hence we conclude that

$$
F\left(U_{\bar{P}}, \tilde{P}\right) \leq \inf _{(U, P)} F(U, P)
$$




\section{$4 \Gamma$-convergence}

The key point of our strategy is to replace the functional (1) by means of more regular functionals by followin a formal $\Gamma$-convergence approach.

Therefore this section is devoted to a very simple presentation of the two results we need: Modica-Mortola's theorem (see [15, 16]) concerning the approximation of the perimeter and De Giorgi's conjecture (see [12]) about the approximation of curvature depending functionals. For the definition of the $\Gamma$-convergence and its main properties we refer the reader to 8, 11 and references therein.

\subsection{Modica Mortola's approach}

Modica-Mortola theorem states that it is possible to approximate, in the $\Gamma$-convergence sense, a perimeter by means of the following sequence of functionals

$$
F_{\varepsilon}^{1}(u):= \begin{cases}\int_{\Omega}\left(\varepsilon|\nabla u|^{2}+\frac{V(u)}{\varepsilon}\right) d x & \text { if } u \in W^{1,2}(\Omega), \\ +\infty & \text { otherwise, }\end{cases}
$$

where $V(u)=u^{2}(1-u)^{2}$ is a double well potential. Besides, since the minimizers of the functional $F_{\varepsilon}^{1}$ may be trivial, some constraint on the functions $u_{\varepsilon}$ must be added. Usually a volume constraint of the type $\int_{\Omega} u d x d y=m$, is assumed.

Let us give an intuitive explanation of such a result. Since $V$ has two absolute minimizers at $u=0,1$, when $\varepsilon$ is small, a local minimizer $u_{\varepsilon}$ is closed to 1 on a part of $\Omega$ and close to 0 on the other part, making a rapid transition of order $\varepsilon$ between 0 and 1 . When $\varepsilon \rightarrow 0$ the transition set shrinks to a set of dimension 1 , so that $u_{\varepsilon}$ goes to a function taking values $u$ into $\{0,1\}$ and the family of functionals $\Gamma$-converges to the measure of the perimeter of the discontinuity set of $u$. Modica-Mortola's Theorem is the following.

Theorem 4.1. The functionals $F_{\varepsilon}^{1}: L^{1}(\Omega) \rightarrow[0,+\infty] \Gamma$-converge with respect to the $L^{1}$-convergence to the following functional

$$
F^{1}(u)= \begin{cases}C_{V} \mathcal{H}^{1}\left(S_{u}\right) & \text { if } u \in\{0,1\} \\ +\infty & \text { otherwise }\end{cases}
$$

where, as usual, $S_{u}$ denotes the set of discontinuities of $u$ and $C_{V}$ is a suitable constant depending on the potential $V$.

\subsection{De Giorgi's conjecture}

The aim of De Giorgi was finding a variational approximation of a curvature depending functional of the type:

$$
F^{2}(D)=\int_{\partial D}\left(1+\kappa^{2}\right) d \mathcal{H}^{1}
$$

${ }^{223}$ where $D$ is a regular set and $k$ is a curvature of its boundary $\partial D$. 
224 Since $\partial D$ can be represented as the discontinuity set of the function $u_{0}=1-\chi_{D}$, 225 by Modica-Mortola's Theorem it follows that there is a sequence of non constant local minimizers such that $u_{\varepsilon} \rightarrow u_{0}$ with respect to the $L^{1}$-convergence such that

$$
\lim _{\varepsilon \rightarrow 0} F_{\varepsilon}^{1}\left(u_{\varepsilon}\right):=C_{V} \mathcal{H}^{1}(\partial D) .
$$

227 Furthermore looking at the Euler-Lagrange equation associated to a contour length term, ${ }_{228}$ yields a contour curvature term $\kappa$, while the Euler-Lagrange equations for the functional $229 \quad F_{\varepsilon}^{1}(u)$ contains a term $2 \varepsilon \Delta u-\frac{V^{\prime}(u)}{\varepsilon}$.

230 Then De Giorgi suggested to approximate the functional $F^{2}$ by adding to Modica231 Mortola's approximating functionals the term

$$
F_{\varepsilon}^{2}(u)=\int_{\Omega}\left(2 \varepsilon \Delta u-\frac{V^{\prime}(u)}{\varepsilon}\right)^{2}\left(\varepsilon|\nabla u|^{2}+\frac{V(u)}{\varepsilon}\right) d x .
$$

In [18 the authors have proven a simplified version of the De Giorgi's conjecture, where the integral above is replaced by the functional

$$
F_{\varepsilon}^{2}(u)=\int_{\Omega}\left(2 \varepsilon \Delta u-\frac{V^{\prime}(u)}{\varepsilon}\right)^{2} d x .
$$


${ }_{232} \quad 5 \quad$ The approximating functionals

248 where

$$
\int_{\Omega \backslash P}|\operatorname{div} U|^{2}+\lambda \int_{\Omega}\left|U-U_{0}\right|^{p}+\mathcal{H}^{0}(P) .
$$

where $U \in L^{p, 2}(\operatorname{div} ; \Omega \backslash P), U_{0} \in \mathcal{D} \mathcal{M}_{\text {loc }}^{p}\left(\mathbb{R}^{2}\right)$ and finally $P$ is an atomic set consisting of a finite number $N$ of points, i.e. $P=\left\{x_{1}, \ldots, x_{N}\right\}$.

As pointed out in the introduction, the first step is to substitute the counting measure $\mathcal{H}^{0}(P)$ with a more treatable term given by:

$$
G_{\beta_{\varepsilon}}(D)=\frac{1}{4 \pi} \int_{\partial D}\left(\frac{1}{\beta_{\varepsilon}}+\beta_{\varepsilon} \kappa^{2}(x)\right) d \mathcal{H}^{1}(x) ;
$$

where $D$ is an union of regular simply connected sets $\left\{D_{i}\right\}$ with $i=1, . ., N$, such that $x_{i} \in D_{i}, D_{i} \bigcap D_{j}=\emptyset$ for $i \neq j . \kappa$ is the curvature of the boundary of the set $D$, the constant $\frac{1}{4 \pi}$ is a normalization factor and $\beta_{\varepsilon}$ is infinitesimal as $\varepsilon \rightarrow 0$.

To understand why we can approximate $\mathcal{H}^{0}(P)$ with $G_{\beta_{\varepsilon}}(D)$ one should note that the solution of the following minimum problem

$$
\min _{D \supset P} G_{\beta_{\varepsilon}}(D)
$$

is given by $D=\bigcup_{i}^{N} B\left(x_{i}, \beta_{\varepsilon}\right)$, where $x_{i}$ are the points of $P$. We give an idea of a possible proof in the case of a single point.

By the Young's inequality we have

$$
G_{\beta_{\varepsilon}}(D) \geq \frac{1}{4 \pi} \int_{\partial D} 2 \kappa d \mathcal{H}^{1}(x)
$$

4 and by applying the Gauss-Bonnet Theorem

$$
G_{\beta_{\varepsilon}}(D) \geq \frac{1}{4 \pi}(2)(2 \pi)=1=\mathcal{H}^{0}(P) .
$$

Finally a simple calculation shows that, if we evaluate the functional $G_{\beta_{\varepsilon}}$ on $B\left(x_{1}, \beta_{\varepsilon}\right)$, we obtain the value 1 , i.e. the number of points in $P$, i.e. $\mathcal{H}^{0}(P)$. The $N$ points case can be recovered with minor changes by the same argument.

For what follows it is convenient to split the functional $G_{\beta_{\varepsilon}}$ in two terms:

$$
G_{\beta_{\varepsilon}}(D)=G_{\beta \varepsilon}^{1}(D)+G_{\beta \varepsilon}^{2}(D)
$$


249

$$
G_{\beta_{\varepsilon}}^{1}(D)=\frac{1}{4 \pi} \int_{\partial D} \frac{1}{\beta \varepsilon} d \mathcal{H}^{1}(x) ;
$$

and

$$
G_{\beta_{\varepsilon}}^{2}(D):=\frac{1}{4 \pi} \int_{\partial D} \beta_{\varepsilon} \kappa^{2}(x) d \mathcal{H}^{1}(x) .
$$

We can write an intermediate approximation of energy (11):

$$
E_{\varepsilon}(U, D)=G_{\beta_{\varepsilon}}^{1}(D)+G_{\beta_{\varepsilon}}^{2}(D)+\int_{\Omega}\left(1-\chi_{D}\right)|\operatorname{div}(U)|^{2}+\lambda \int_{\Omega}\left|U-U_{0}\right|^{p} .
$$

The advantage of such a formulation is that we know how to provide a variational approximation of the perimeter measure $H^{1}\lfloor\partial D$. Following Modica-Mortola's approach such an approximation can be obtained by using the following measure:

$$
\mu_{\varepsilon}(w, \nabla w) d x=\left(\varepsilon|\nabla w|^{2}+\frac{V(w)}{\varepsilon}\right) d x
$$

where $V(w)=w^{2}(1-w)^{2}$ is a double well functional.

Next step is expressing the curvature term by means of the function $w$. Thanks to the simplified version of the De Giorgi's conjecture we can replace the term $\kappa$ by the term $2 \varepsilon \Delta w-\frac{V^{\prime}(w)}{\varepsilon}$.

So that we can formally write the complete approximating functional:

$$
\begin{aligned}
\Phi_{\varepsilon}(U, w): & =\int_{\Omega} w^{2}|\operatorname{div}(U)|^{2} d x+\frac{1}{4 \pi} \int_{\Omega} \beta_{\varepsilon}\left(2 \varepsilon \Delta w-\frac{V^{\prime}(w)}{\varepsilon}\right)^{2} d x+\frac{1}{\beta_{\varepsilon}} \int_{\Omega} \mu_{\varepsilon}(w, \nabla w) d x \\
& +\lambda \int_{\Omega}\left|U-U_{0}\right|^{p} d x+\frac{2}{\mu_{\varepsilon}} \int_{\Omega}(1-w)^{2} d x
\end{aligned}
$$

where $U \in L^{p, 2}(\operatorname{div} ; \Omega)$ is equal to 0 on the $\partial \Omega$ and $w$ is smooth function equal to 1 on the boundary, i.e. $1-w \in C_{0}^{\infty}(\Omega), \mu_{\varepsilon} \rightarrow 0$ when $\varepsilon$ goes to 0 . The last integral is a penalization term which prevents $w_{\varepsilon}$ from converging to the function constantly equal to 0 as $\varepsilon \rightarrow 0$.

Then if $\left(U_{\varepsilon}, w_{\varepsilon}\right)$ is a minimizing sequence of $\Phi_{\varepsilon}$, then $w_{\varepsilon}$ must be very close to the values 1 when $\varepsilon$ goes to 0 , since the double well potential is positive except for $w_{\varepsilon}=0,1$ and $w$ must be equal to 1 on $\partial \Omega$. On the other hand, near the points where the divergence is very big $w_{\varepsilon}$ must be close to 0 . Besides when $\varepsilon \rightarrow 0, \beta_{\varepsilon} \rightarrow 0$ goes to 0 as well, so that the singular set $D$ is given by an union of balls of a small radius $\beta_{\varepsilon}$.

Therefore, while the functions $U_{\varepsilon}$ approximate a minimizer $U$ of the original functional, the level set $\left\{w_{\varepsilon}=0\right\}$ approximate the original singular set $P$.

Remark 5.1. We point out that the $\Gamma$-convergence result is not proved in this paper, but only conjectured. A complete proof of the $\Gamma$-convergence result and the equicoerciveness of the sequence $\Phi_{\varepsilon}$, in the particular case where the vector field $U$ is a gradient, has been provided by the first and third author in [6]. 
The first variation of this functional leads to the following gradient flow system

$$
\begin{aligned}
& \frac{\partial U}{\partial t}=2 \nabla\left(w^{2} \operatorname{div} U\right)+\lambda p\left|U-U_{0}\right|^{p-2}\left(U-U_{0}\right) \\
& \frac{\partial w}{\partial t}=-4 \frac{\Delta h}{\beta_{\varepsilon}}+\beta_{\varepsilon} h+\frac{2}{\varepsilon^{2}} \frac{1}{\beta_{\varepsilon}} V^{\prime \prime}(w) h-2 w|\operatorname{div} U|^{2}+2 \frac{1}{\mu_{\varepsilon}}(1-w),
\end{aligned}
$$

${ }_{273}$ where $h$ is given by the equation

$$
h=2 \varepsilon \Delta w-\frac{1}{\varepsilon} V^{\prime}(w) .
$$




\section{${ }_{274} 6$ Detection}

275 In our model the image contains an atomic Radon measure. Thus, in order to find an initial 276 vector field which copies the singularities of the initial image, we use the gradient of the 277 solution of the following Dirichlet problem:

$$
\begin{cases}\Delta f=I & \text { on } \Omega \\ f=0 & \text { on } \partial \Omega .\end{cases}
$$

\subsection{Discretization}

The image is an array of size $N^{2}$. We endowed the space $R^{N \times N}$ with the standard scalar product and standard norm. The gradient $\nabla I \in\left(R^{N \times N}\right) \times\left(R^{N \times N}\right)$ is given by:

$$
(\nabla I)_{i, j}=\left((\nabla I)_{i, j}^{1},(\nabla I)_{i, j}^{2}\right)
$$

where

$$
\begin{aligned}
& (\nabla I)_{i, j}^{1}= \begin{cases}I_{i+1, j}-I_{i, j} & \text { if } i<N \\
0 & \text { if } i=N\end{cases} \\
& (\nabla I)_{i, j}^{2}= \begin{cases}I_{i, j+1}-I_{i, j} & \text { if } j<N \\
0 & \text { if } j=0 .\end{cases}
\end{aligned}
$$

We also introduce the discrete version of the divergence operator simply defined as the adjoint operator of the gradient: $\operatorname{div}=-\nabla^{*}$. More in details if $v \in\left(R^{N \times N}\right) \times\left(R^{N \times N}\right)$, we have 


$$
(\operatorname{div} v)_{i, j}= \begin{cases}v_{i, j}^{1}+v_{i, j}^{2} & \text { if } i, j=1 \\ v_{i, j}^{1}+v_{i, j}^{2}-v_{i-1, j}^{2} & \text { if } i=1,1<j<N \\ v_{i, j}^{1}-v_{i-1, j}^{1}+v_{i, j}^{2}-v_{i-1, j}^{2} & \text { if } 1<i<N, 1<j<N \\ -v_{i-1, j}^{1}+v_{i, j}^{2}-v_{i-1, j}^{2} & \text { if } i=N, 1<j<N \\ v_{i, j}^{1}-v_{i-1, j}^{1}+v_{i, j}^{2} & \text { if } 1<i<N, j=1 \\ v_{i, j}^{1}-v_{i-1, j}^{1}-v_{i-1, j}^{2} & \text { if } 1<i<N, j=N \\ -\left(v_{i-1, j}^{1}+v_{i-1, j}^{2}\right) & \text { if } i, j=N .\end{cases}
$$

Then we can define the discrete version of the Laplacian operator as $\Delta I=\operatorname{div}(\nabla I)$.

\subsection{Discretization in time}

We simply replace $\frac{\partial U}{\partial t}$ and $\frac{\partial w}{\partial t}$ by $\frac{U_{i, j}^{n+1}-U_{i, j}^{n}}{\delta t}$ and $\frac{w_{i, j}^{n+1}-w_{i, j}^{n}}{\delta t}$ respectively. Then we write system (14) in the form (for simplicity we omit the dependence on $\varepsilon$ )

$$
\left\{\begin{array}{l}
U_{1}^{n+1}=-\delta t \Phi_{U_{1}}\left(U_{n}, w_{n}\right) \\
U_{2}^{n+1}=-\delta t \Phi_{U_{2}}\left(U_{n}, w_{n}\right) \\
w^{n+1}=-\delta t \Phi_{w}\left(U_{n}, w_{n}\right) .
\end{array}\right.
$$

We initialize our algorithm with $U(0)=\nabla f$, where $f$ is the solution of problem (15). To do this, we need to solve a Dirichlet problem with data measure $I$, therefore we regularize the image by convolution with a Gaussian kernel $G_{\sigma}$ with very small $\sigma$ and then we solve, by classical finite differences method, the problem:

$$
\begin{cases}\Delta f=I_{\sigma} & \text { on } \Omega \\ f=0 & \partial \Omega,\end{cases}
$$

where $I_{\sigma}=I * G_{\sigma}$.

To initialize our algorithm, we need of an initial guess on $w$. So we choose $w(0)=1$. 


\section{Computer examples}

\subsection{Parameter settings}

Before running our algorithm all the parameters have to be fixed. The most important are $\varepsilon, \beta_{\varepsilon}$ and $\mu_{\varepsilon}$, which govern the set $D$ approximating points we want to detect. Those parameters are related by the conditions $\lim _{\varepsilon \rightarrow 0} \frac{\varepsilon|\log (\varepsilon)|}{\beta_{\varepsilon}}=0, \lim _{\varepsilon \rightarrow 0} \frac{\beta_{\varepsilon}}{\mu_{\varepsilon}}=0$. Furthermore, since the mesh gride size is 1 and $\beta_{\varepsilon}$ gives the radius of a ball centered in the singular point we want to detect, from a discrete point of view the smallest value we can take is $\frac{\sqrt{2}}{2}$. Then we use the values 0.1 for $\varepsilon, 0.7$ for $\beta_{\varepsilon}$, and 0.8 for $\mu_{\varepsilon}$. As exponent $p$ of the discrepancy term we always take $p=1.5$

Concerning the parameter $\lambda$ we mainly used the value $\lambda=0.1$, in order to force the algorithm to regularize as much as possible the initial data $U_{0}$.

Since we deal with small values of $\varepsilon$, in order to have some stability, we must take a small discretization time step. Practically we mainly used the value $\delta t=1 \times 10^{-6}$.

Concerning the stopping criterion we iterate the algorithm until $\max \left\{\frac{\left\|U_{1}^{n+1}-U_{1}^{n}\right\|_{1}}{\left\|U_{1}^{n}\right\|_{1}}, \frac{\left\|U_{2}^{n+1}-U_{2}^{n}\right\|_{1}}{\left\|U_{2}^{n}\right\|_{1}}, \frac{\left\|w^{n+1}-w^{n}\right\|_{1}}{\left\|w^{n}\right\|_{1}}\right\}$ $\leq 1 \times 10^{-} 2$.

In all the computer examples the points are detected by means of the function $w_{\varepsilon}$. We display the level-set $\left\{w_{\varepsilon} \simeq 0\right\}$.

\subsection{Commentaries}

The figure 1 shows how resistant to the noise our model is. When the noise is larger the parameter $\varepsilon$ must be as closer as possible to the ideal value 0 . Besides it is possible to see that for small values of $\varepsilon$ our detection is very fine according to the continuous setting. More in details in the first row we display the initial image obtained by adding a Gaussian noise to a binary image of five points. The second row shows the behavior of $w_{\varepsilon}$ for small values of $\varepsilon$ and $\beta_{\varepsilon}$.

Looking at the histograms of the gray level of $I$ and $w_{\varepsilon}$, one can see that it is easier fixing a threshold value starting from the function $w_{\varepsilon}$ than from the initial image $I$. In the last row we display the set $\left\{w_{\varepsilon} \simeq 0\right\}$ obtained by plotting the set $\left\{w_{\varepsilon} \leq \alpha\right\}$ with threshold value $\alpha=0.5$.

In figure 2 we test our algorithm on curves and points at the same time. In the first row we have a sequence of points and a curve with boundary inside $\Omega$. In the second row we display the function $w_{\varepsilon}$ and the level set $\left\{w_{\varepsilon} \simeq 0\right\}$ once again obtained by fixing a threshold value $\alpha=0.5$. The result is that, as desired, our algorithm is capable of eliminating the curve from the initial image. According to the continuous setting when $\varepsilon$ takes values close to 0 the approximating energy (13) behaves similarly to the limit energy (1), so that the presence of the curve is penalized in the minimization process. Then the set $\left\{w_{\varepsilon} \cong 0\right\}$ contains nothing else but points.

Finally in figure (a),(b),(c) and (d) we deal with a biological image. Our task is catching the finest structure present in the image. In figure (d) the isolated points are quite 
well detected, while the branches the cellule are not. Nevertheless due to the small time discretization step the computation time is quite large. To test the image in (a) of size $500 \times 500$, our algorithm takes 100 iterations and about $7 \mathrm{~mm}$. Certainly the algorithm can be accelerated by using more sophisticated techniques such as multigrid methods. Such a faster algorithm is the subject of our current investigation.

\begin{tabular}{|c|c|c|}
\hline Original image $I$ & Noisy image PSNR $=5.5 \mathrm{Db}$ & Noisy image PSNR $=5.5 \mathrm{Db}$ \\
\hline 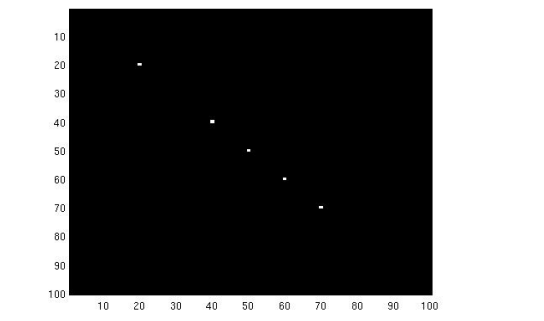 & 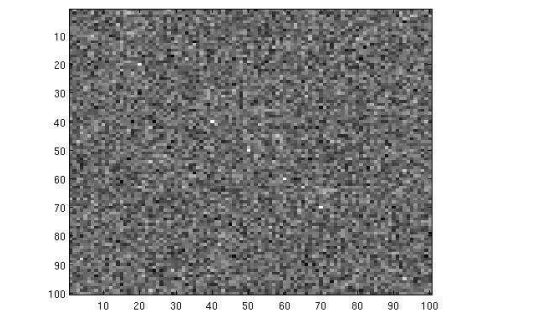 & 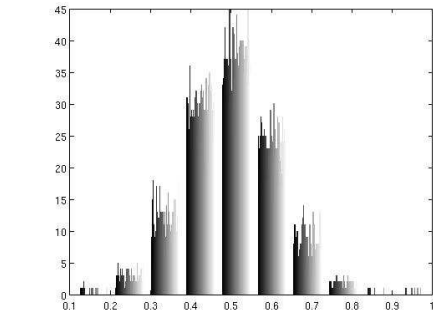 \\
\hline The function $w_{\varepsilon}\left(\varepsilon=0.1, \beta_{\varepsilon}=0.7\right)$ & The function $w_{\varepsilon}\left(\varepsilon=0.1, \beta_{\varepsilon}=0.7\right)$ & $\overline{\overline{w_{\varepsilon}} \simeq 0\left(\varepsilon=0.1, \beta_{\varepsilon}=0.7\right)}$ \\
\hline${ }_{100}{ }_{100}^{20}$ & 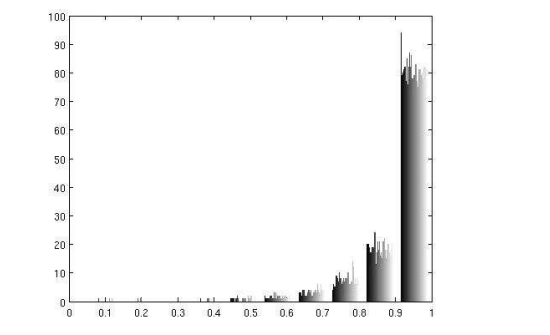 & 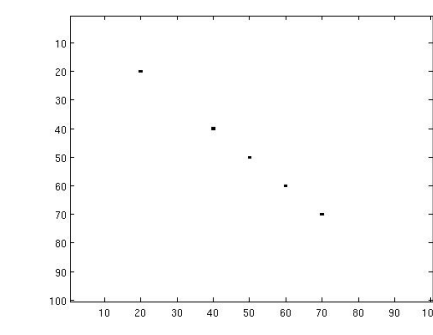 \\
\hline
\end{tabular}

Figure 1: Synthetic image: we test our algorithm on noisy images. When the parameters $\varepsilon$ and $\beta_{\varepsilon}$ are small as much as possible the detection is finer. The detection is refined by fixing a threshold value $\alpha$ for the function $w_{\varepsilon}$ 


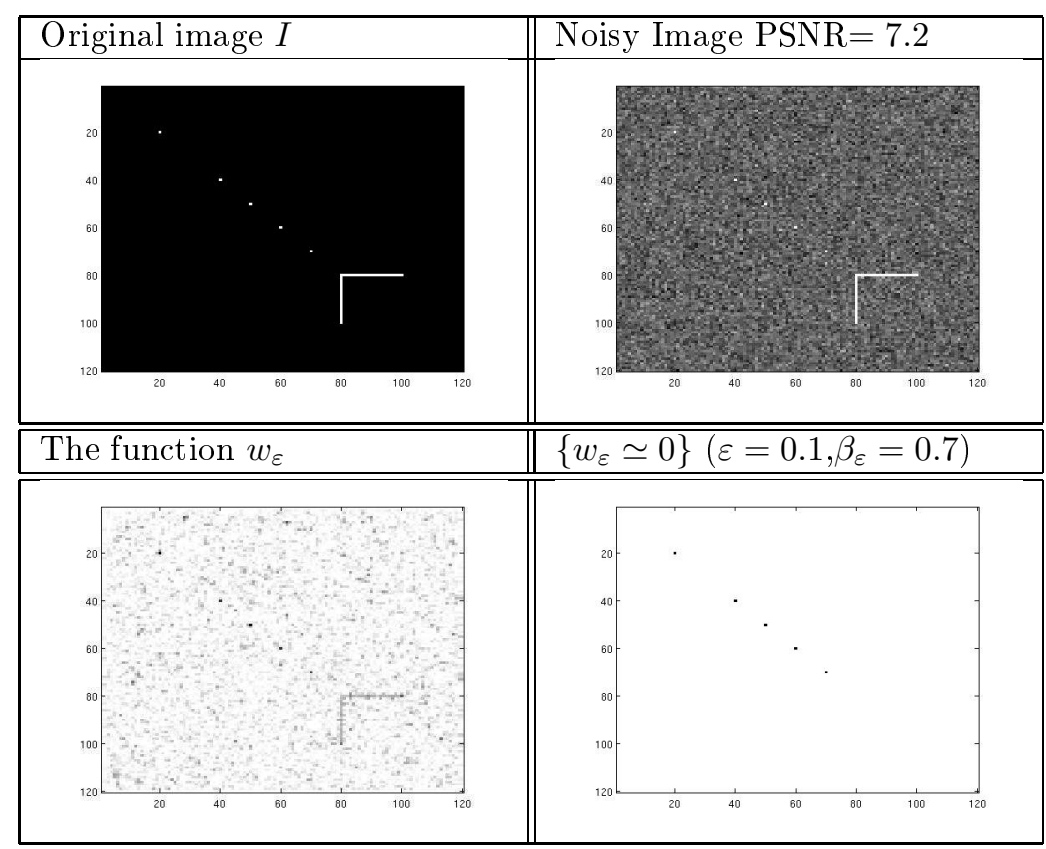

Figure 2: Synthetic image: curve points and noise are present in the initial image. As expected our method is capable of removing the curve from the image. 


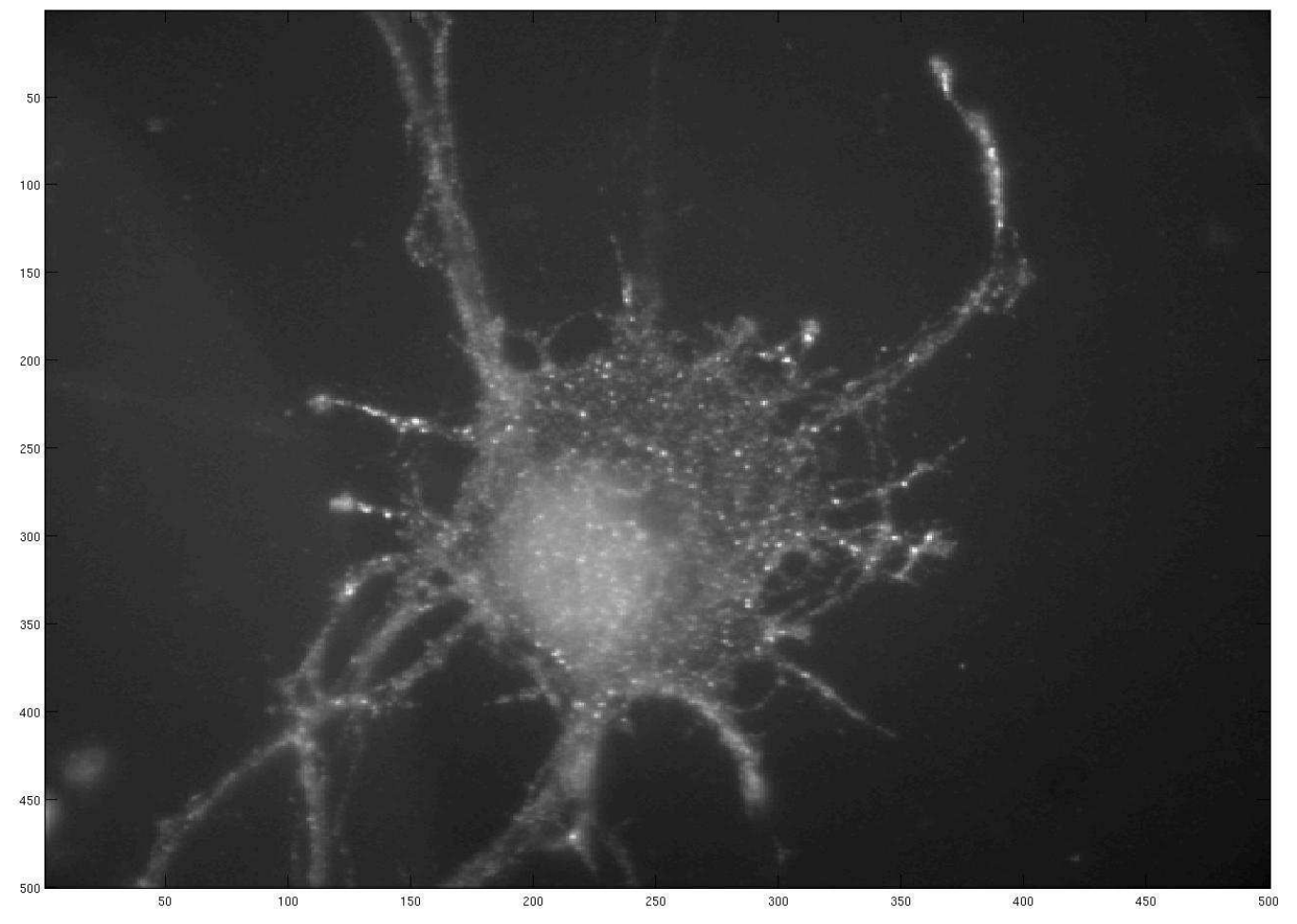

(a) Original Image

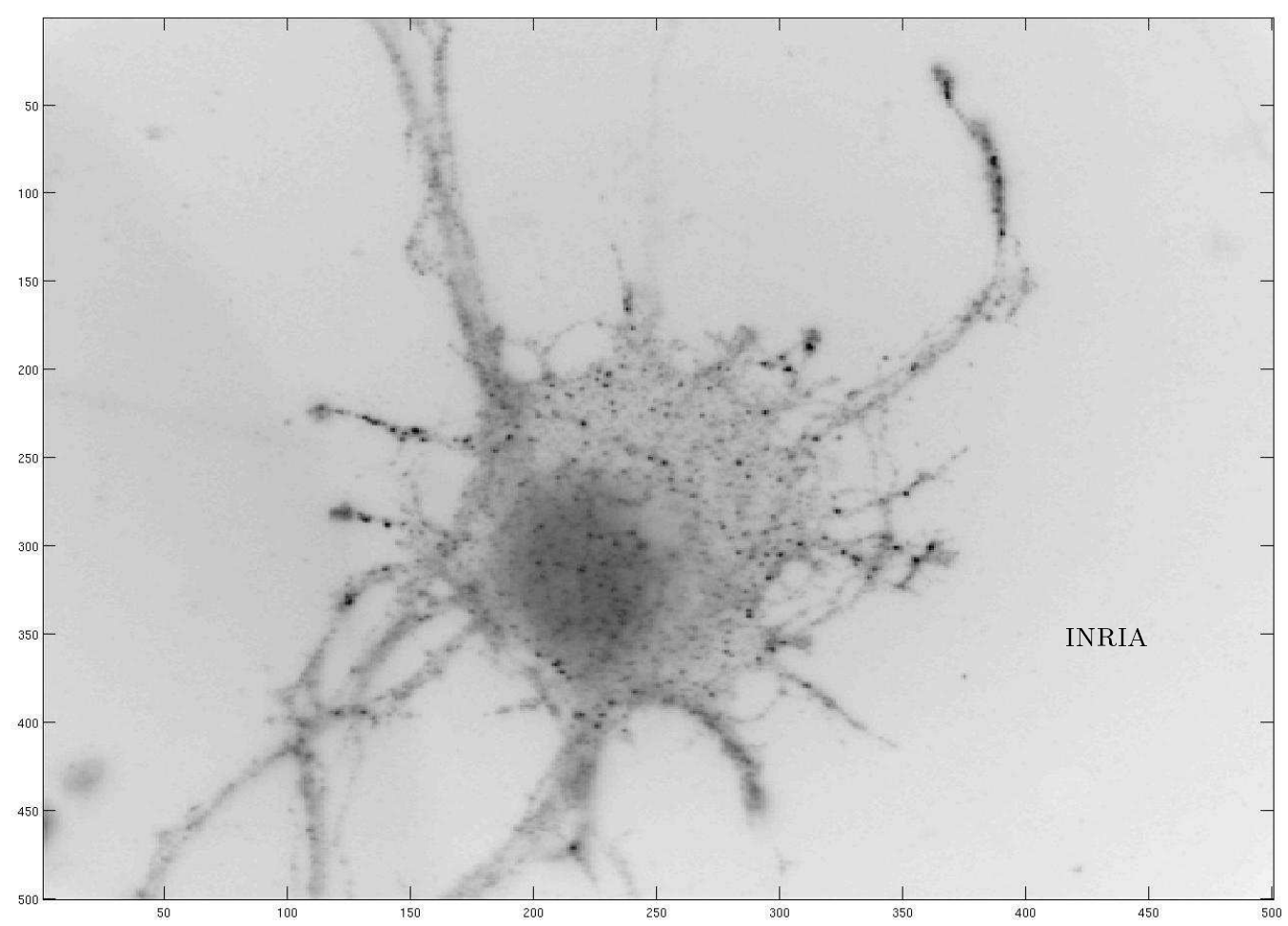

(b) Superposition of the original image with the set $\left\{w_{\varepsilon} \simeq 0\right\}\left(\varepsilon=0.1, \beta_{\varepsilon}=0.7\right)$ 


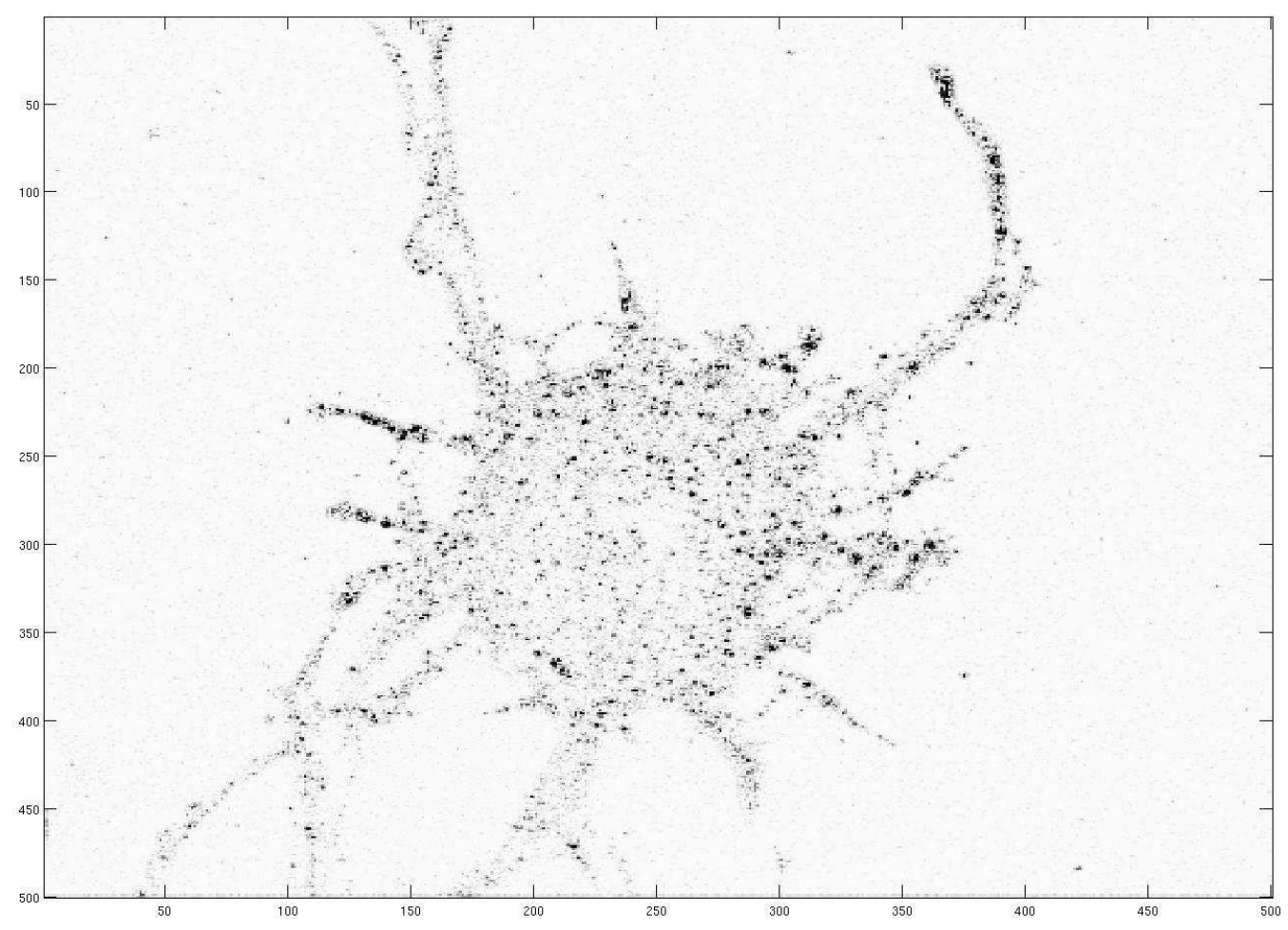

(c) The function $w_{\varepsilon}\left(\varepsilon=0.1, \beta_{\varepsilon}=0.7\right)$

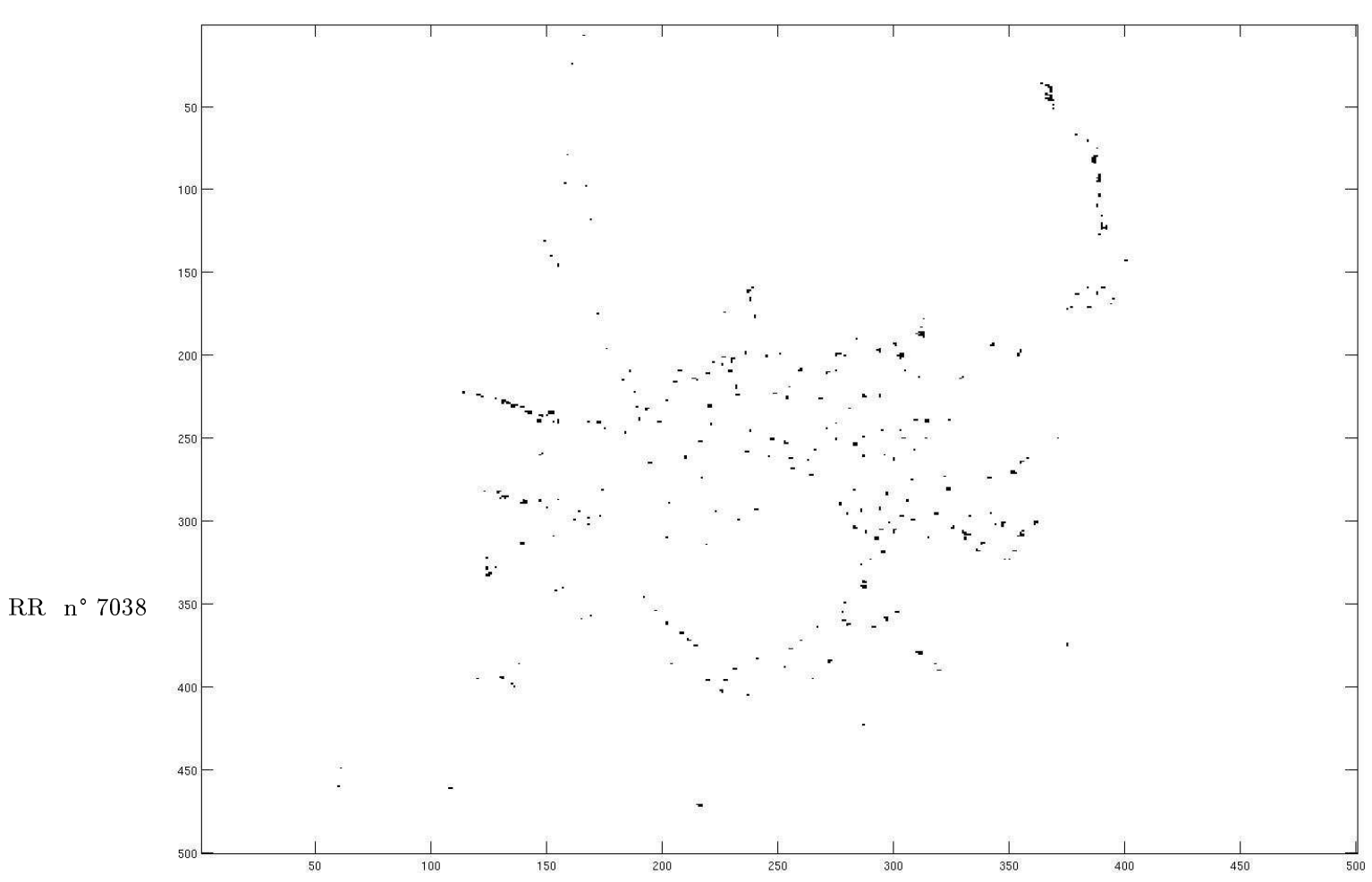

(d) the set $\left\{w_{\varepsilon} \simeq 0\right\}\left(\varepsilon=0.1, \beta_{\varepsilon}=0.7\right)$ 


\section{${ }_{343} 8$ Conclusion}

344 In this work, a new variational method for spot detection in biological images has been

${ }_{345}$ proposed and tested. We emphasize that, according to our knolewdge, this is the first

346 method which makes possible isolating the spots from a filament in the observed image.

347 Moreover it also permits in a noisy image to fix a threshold value in a simple and direct way.

348 Moreover we believe that a suitable generalization of this method for the detection of spots

349 and even filaments in 3-D biological images can be provided. This is a subject of our current

350 investigation. Certainly there are many rooms for improvement both from a theoretical and

${ }_{351}$ numerical point of view such as a deep investigation of the $\Gamma$-convergence approximation, as

352 a well as a significant acceleration of the algorithm. 


\section{References}

[1] L.Ambrosio, N.Fusco, D.Pallara. Functions of bounded variation and free discontinuity problems. Oxford University Press (2000).

[2] L.Ambrosio, V.M.Tortorelli. Approximation of functionals depending on jumps by elliptic functionals via $\Gamma$-convergence. Communic. Pure Appl. Math 43 (1990), 999-1036.

[3] L.Ambrosio, V.M.Tortorelli. Approximation of functionals depending on jumps by quadratic, elliptic functionals via $\Gamma$-convergence. Boll.Un. Mat. Ital. 6-B(1992), 105-123.

[4] G.Anzellotti Pairings between measures and bounded functions and compensated compactness. Ann. Mat. Pura Appl. 135 (1983), 293-318.

[5] G.Aubert, J. Aujol, L.Blanc-Feraud Detecting Codimension-Two Objects in an image with Ginzurbg-Landau Models. International Journal of Computer Vision 65 (2005), 2942.

[6] G.Aubert, D.Graziani Variational approximation for detecting points like-target problem in 2-D biological images submitted to ESAIM: COCV. Preprint available on http://wwwsop.inria.fr/en/publications.php.

[7] F. Bethuel, H. Brezis and F. Hélein Ginzburg-Landau Vortices. Birkäuser, Boston (1994).

[8] A.Braides. $\Gamma$-convergence for beginners. Oxford University Press, New york (2000).

[9] A.Braides, A.Malchiodi Curvature Theory of Boundary phases: the two dimensional case. Interfaces Free. Bound. 4 (2002), 345-370.

[10] A.Braides, R.March. Approximation by $\Gamma$-convergence of a curvature-depending functional in Visual Reconstruction Comm. Pure Appl. Math. 59 (2006), 71-121.

[11] G.Dal Maso. Introduction to Г-convergence. Birkhäuser, Boston(1993).

[12] E.De Giorgi Some Remarks on $\Gamma$-convergence and least square methods, in Composite Media and Homogenization Theory (G. Dal Maso and G.F.Dell'Antonio eds.), Birkhauser, Boston, 1991, 135-142.

[13] E.De Giorgi, T.Franzoni. Su un tipo di convergenza variazionale. Atti Accad. Naz. Lincei Rend. Cl. Sci. Mat. Natur. 58 (1975), 842-850.

[14] E.De Giorgi, T.Franzoni. Su un tipo di convergenza variazionale. Rend. Sem. Mat. Brescia 3 (1979), 63-101.

[15] L.Modica, S.Mortola. Un esempio di Г-convergenza. Boll.Un. Mat. Ital. 14-B(1977), $285-299$. 
384

385

386

[16] L.Modica. The gradient theory of phase transitions and the minimal interface criterion. Arch. Rational Mech. Anal. 98 (1987), 123-142.

[17] M.Morel, Solimini Variational models in image segmentation, Birkäuser, 1994.

[18] M.Röger, R.Shätzle. On a modified conjecture of De Giorgi. Math. Zeitschrift. 254 (2006), 675-714

[19] G.Stampacchia Le problème de Dirichlet pour les equations elliptiques du seconde ordre à coefficients discontinuous. Ann. Inst. Fourier (Grenoble), 15 (1965), 180-258.

[20] A.Witkin, D.Terzopoulos, M.Kass. Signal mathcing trough scale space International Journal of Computer Vision 12 (1987), 133-144. 


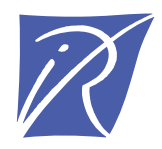

\section{Unité de recherche INRIA Sophia Antipolis 2004, route des Lucioles - BP 93 - 06902 Sophia Antipolis Cedex (France)}

Unité de recherche INRIA Futurs : Parc Club Orsay Université - ZAC des Vignes 4, rue Jacques Monod - 91893 ORSAY Cedex (France) Unité de recherche INRIA Lorraine : LORIA, Technopôle de Nancy-Brabois - Campus scientifique 615, rue du Jardin Botanique - BP 101 - 54602 Villers-lès-Nancy Cedex (France)

Unité de recherche INRIA Rennes : IRISA, Campus universitaire de Beaulieu - 35042 Rennes Cedex (France)

Unité de recherche INRIA Rhône-Alpes : 655, avenue de l'Europe - 38334 Montbonnot Saint-Ismier (France)

Unité de recherche INRIA Rocquencourt : Domaine de Voluceau - Rocquencourt - BP 105 - 78153 Le Chesnay Cedex (France) 